\title{
Design in 3D: A computational fashion design protocol
}

Introduction

Computational fashion design research is an important area of inquiry for both design

4 researchers in the apparel industry as well as academic community. Throughout the last 50

5 years, clothing design and construction methods have already undergone significant changes to

6 become a fully automated, computer-aided process (Lee, 2014; Loker, Ashdown, \& Carnrite,

7 2008). The use of computer-aided-design (CAD) software offers many new possibilities and it is

8 critical to reconcile the use of technology and traditional garment construction methods for

9 wide-scale implementation in the design process (Lee, 2014). In this paper, the definition of

10 parametric and algorithmic design refers to processes based in parametric mathematics and

11 manipulated through the particularity of an algorithm. In what follows, the use of algorithms and

12 computational design methods were explored to add to the growing body of literature on the use

13 of digital technology in computer-aided fashion design. While parametric design is not new,

14 computational programs have enabled a wider retinue of options to build and design with greater

15 dexterity and reproducibility.

16 The purpose of this study was to create a corset - understructure as well as fabric

17 covering - using only computational, 3D approaches to fashion design. The process incorporated

18 3D body scan data, parametric methods for the 3D printed design, and algorithmic methods for

19 the automated, custom-fit fabric pattern. In what follows, researchers situate the relevance of this

20 project and provide an adapted framework to guide future methods-based design scholarship.

\section{Design Framework}

Elizabeth Bye’s paper, “A Direction for Clothing and Textile Research” (2010) provided

23 much-needed context and infrastructure for design-based research in the field of apparel design. 
24 Inspired by Bye's 'research-through-practice' framework (2010, p. 208), a method or procedure-

25 based design framework is proposed in this paper to provide context and precipitate

26 conversations among designers about nascent techniques and construction methods, where

27 innovation and reproducibility are the final contributions to the larger apparel design field. The

28 orbital model presented in Figure 1 places the methods at the nucleus of the diagram where

29 inquiry, contextual review, practice and reflection, analysis, and the final prototype or artifact,

30 are all reflexively informed by modifications to the algorithms in the computational design

31 protocol. While this project used a computational approach, the framework below acknowledges

32 the significance of procedure and technique in many forms of design research.

33 Design research is often characterized by the blurring of the distinction between research

34 and design which ultimately becomes a circular, iterative process (Edelson, 2002). In keeping

35 with the circular nature of the design process and the three-dimensional ethos of this paper,

36 Bye's framework was modified to illustrate the procedure in this design project wherein the

37 protocol is located at the center of the sphere because the construction of a protocol/process is

38 both the nucleus for the research as well as the final outcome (Figure 1). 


\section{Methods-Based Design Scholarship Framework}

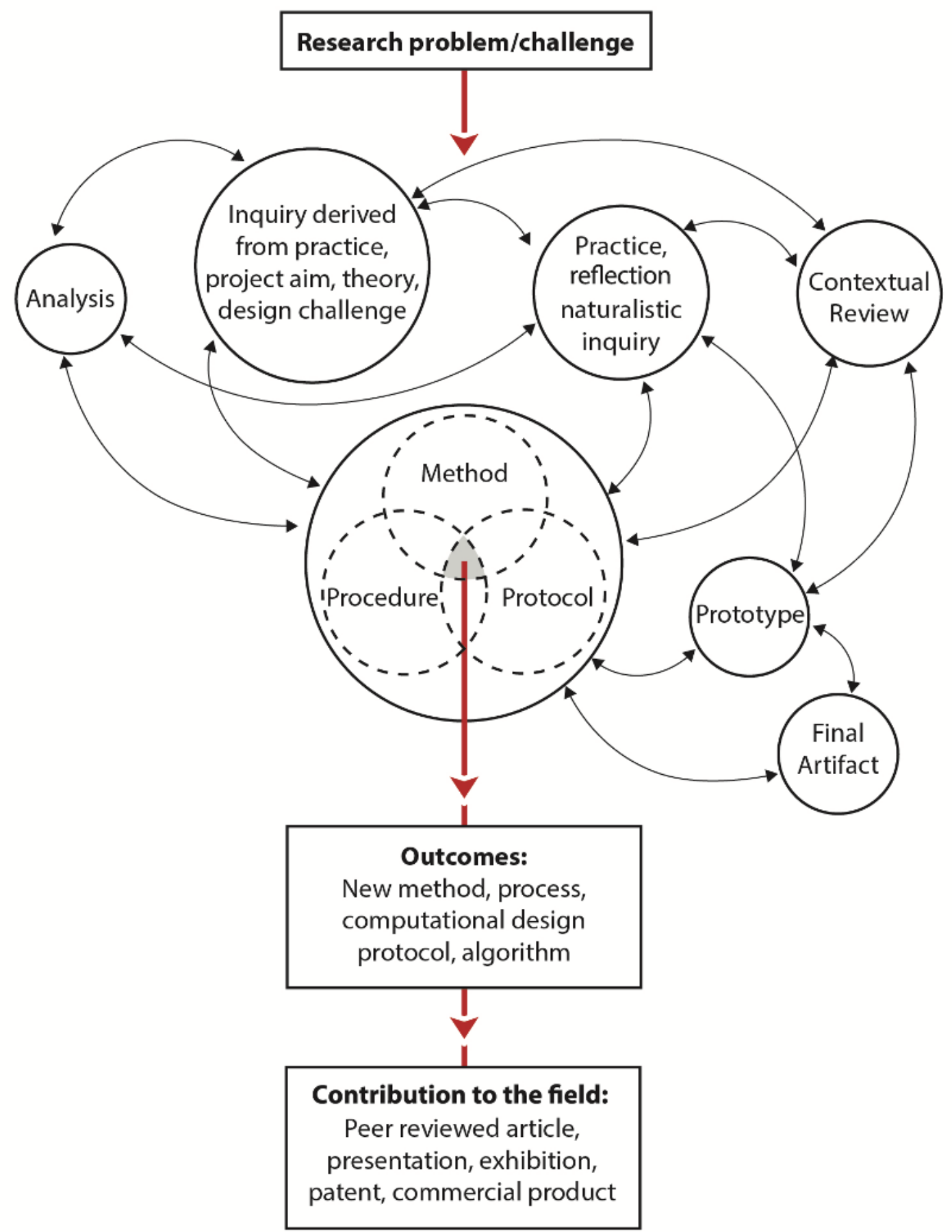

Figure 1. Methods-based design scholarship framework. 


\section{Situating 3D Computational Fashion Design}

42 In the late 1980s and early 1990s, research in sizing and fit used somatograms to

43 experiment with accuracy in moving from three-dimensional forms to two-dimensional

44 computer-generated patterns (Winakor et al., 1990). Because the widescale adoption of CAD

45 programming was relatively new, researchers explored the use of geometric pattern classification

46 through body measurements, to assess whether or not computer-generated patterns were as easily

47 altered as traditional pattern drafting techniques. Later, as CAD programming became more

48 widely adopted, researchers in the mid-2000s explored the use of 3D body scan data to create a

49 basic skirt patterns (Griffey and Ashdown, 2006), the relationship between body shape and ease

50 value in trousers (Petrova and Ashdown, 2008), and automatically place a side seam using only

51 scan data (Ashdown et al., 2008). The increased availability of CAD programing enabled

52 researchers to examine $2 \mathrm{D}$ and $3 \mathrm{D}$ shape construction in conjunction with traditional draping

53 techniques (Townsend, 2004; Bye, 2010). Song and Ashdown (2010) conducted an exploratory

54 study into the use of body scan data in virtual fit assessment analysis and later, they similarly

55 explored the use of virtual fit software with respect to pants (Song and Ashdown, 2015). Song

56 and Ashdown $(2010,2015)$ showed the ways in which 3D simulation enables the designer to

57 zoom, rotate, and analyze fit issues in a virtual format, techniques which if perfected, would

58 minimize the need for muslin fittings and increase production efficiency. As such, apparel

59 product design companies have largely adopted the use of virtual simulation software to save

60 both time and money (Lee, 2014). Apparel industry adoption of virtual fit analysis software has

61 also been a subject of ongoing research in consumer behavior, fit analysis, and marketing (Beck

62 \& Crié, 2018; Kim \& Forsythe, 2008; Lee \& Park, 2017; Lin \& Wang, 2016; Merle, Senecal, \&

63 St-Onge, 2012). 
Sungmin Kim's (2015) paper on the use of parametric design methods to analyze bodily

65 concavity and convexity, offered insight on the interplay between parametric design in

66 conjunction with 3D body scan data sets. In the fashion industry and museum world, 3D printed

67 garments have been shown on the runways and have subsequently been accessioned into

68 museum fashion collections. Fashion designer Iris van Herpen's iconic 3D printed designs are

69 emblematic of the use of parametrics on the fashion runway. Referred to as "haute couture's

70 chief scientist and perhaps also its leading futurist" Van Herpen's recent Fall 2018 show titled

71 Syntopia was called a merging of biology and technology (Verner, 2018). Merging traditional

72 tailoring techniques and the latest in computer aided design, Van Herpen is known to start from a

73 sketch after which she works with an architect to realize the 3D structure. Syntopia was just one

74 example of innovation driven design and construction methods which rely on parametrics to

75 capture the marriage between analogue and machine techniques.

Methods and Computational Design Protocol

The 3D computational methods used in this study enabled a new way of rendering the body-

to-pattern relationship through the use of multiple software platforms. Drawing on a $19^{\text {th }}$ century

79 historical garment - the corset - computational design methods were used to similarly

80 manipulate 3D proportion and form using a structured undergarment and custom fit pattern for

81 the fabric layer. Because the corset typifies an early synthesis of clothing and technology, it was

82 chosen as the starting point for this research. The 3D printed structure was created using

83 parametric design techniques while the design of the fabric layer used an integrated computer

84 algorithm which automatically generated snug-fit pattern pieces for the 3D scanned body on

85 which the 3D printed structure was based. The automated operation of pattern generation took

86 one click, in a timeframe of less than two seconds. It has been shown that 3D body scanning 
87 offers exponentially more data points which provide a more accurate account of an individual

88 body (Ashdown and Loker, 2010; Daanen and Ter Haar, 2013; McKinney et al., 2017; Poole and

89 Shvartzberg, 2015).

90 The individual 3D body scan used in this study originally had 780,381 points and was

91 randomly selected from the CAESAR® (Civilian American and European Surface

92 Anthropometry Resource Project) database. Four computer software/programs were used to

93 process the scan: Geomagic ${ }^{\circledR}$ and Rhinoceros ${ }^{\circledR}$ in creating the $3 \mathrm{D}$ corset structure; Matlab ${ }^{\circledR}$ and

94 CLO3D to automatically generate and modify the customized pattern pieces. The fit of the raw

95 patterns was verified in CLO3D, a virtual garment simulation software. CLO3D was chosen as

96 the visualization software because it was thought to be more user-friendly as compared to the 3D

97 simulation function in Optitex ${ }^{\mathrm{TM}}$. Figure 2 features the work flow of the design protocol and

98 what follows is a more detailed description of the procedure. 




99

100 Figure 2: Work flow of the design protocol.

101 The 3D Printed Corset

102 The construction of the corset structure began by using Geomagic $®$, an engineering

103 software which enables 3D scan data to be processed and analyzed in CAD software. This

104 software was used because of its ease in rendering mesh shapes as they translate into solid forms. 
105 The scan selected for this project was imported into Geomagic $®$, where the head, the neck and 106 the limbs of the scan were removed, keeping only the torso as this research focused only on the 107 creation of a wearable garment for this area of the body. Due to the fact that participants of the 108 CAESAR Project were scanned facing a $45^{\circ}$ angle to the true front, all CAESAR scans require 109 rotation. In this case, rotation was also done in Geomagic®. Functions such as "Mesh Doctor" 110 and "Fill Holes", both built-in applications or plug-ins, were used to enhance the quality of mesh 111 on the scan surface thereby improving the quality of the 3D printed object. It was necessary to 112 wrap the mesh into a smooth surface to be able to work in most CAD software. To these ends, 113 the following steps were taken: Contour detection and manual edition, patch and grid 114 construction, fitting of surfaces and conversion to CAD file (Figure 3). The exported file was 115 in .igs format and was ready to be imported into Rhino® for parametric design (Figure 4a).
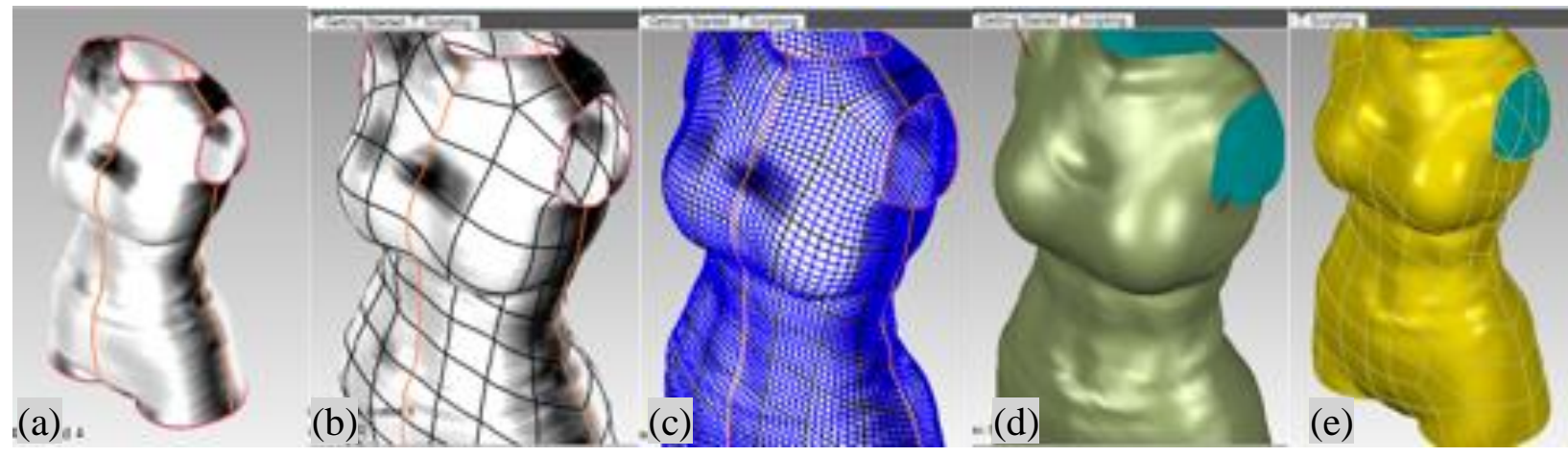

117 Figure 3. Processing of the 3D body scan in Geomagic $®$ : (a). Contour detection. (b). Creation of patches. (c). Creation of grids. (d). Fitting of new surfaces. (e). Conversion to CAD file.

121 A Grasshopper program — a visual programming language that runs within Rhino®—was written

122 to create a multi-hexagon structure that fits to the new surface formed by the contour lines

123 (Figure 4c). Transforming 1D hexagonal line segments into 3D pillars was attempted; however,

124 due to software limitations it was not possible to join the pillars. In addition, there were small

125 gaps at the edges of the pillar connections. These two factors increased the likelihood of 
126 significant problems during the 3D printing process. Therefore, the Grasshopper program was 127 modified to create a 2D surface composed of the same hexagons, only this time the sides of the 128 hexagons included widths (Figure 4d). Then the shape of the structure was modified aiming for 129 asymmetrical design elements. Multiple trials were undertaken during this process before 130 finalizing the object shown in Figure 4d.



132 Figure 4. Creation of a surface with hexagon hollows in Rhino®: (a). Initial import. (b). 133 Reconstructed horizontal contour lines. (c). 1D Multi-hexagon structure. (d). 2D Multi-hexagon 134 structure.

Once the necessary contours that modify the shape of the structure for wearability were

137 achieved, drill holes were added at center front, center back, and down both the proper left and 138 right seams for lacing; the aim was to ultimately separate the structure into four separate

139 quadrants for 3D printing (Figure 5). To add the drill holes for lacing, the hexagonal structure 140 along the computer-generated seams were closed as directly cutting holes on the hexagon edges

141 reduced the strength of the structure, making it very likely to break under pressure. Moreover, a

142 few hexagon hollows at the bust area were also closed in consideration of modesty (Figure 5a).

143 At this stage, there was a technical challenge in transforming the two-dimensional surface into

144 three dimensions (i.e., the addition of thickness added to the hexagonal geometry), due to the 145 excess number of faces on the surface area. This was resolved by first turning the parametric 
146 surface into a mesh surface (Figure 5b). Figure 5 also shows the initial design aesthetic designed 147 via 3D modeling in Rhino®. The file was then exported into .stl format for 3D printing. (a)
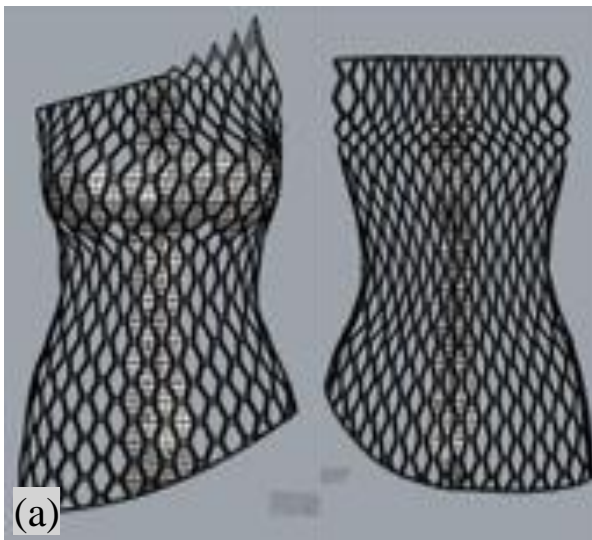

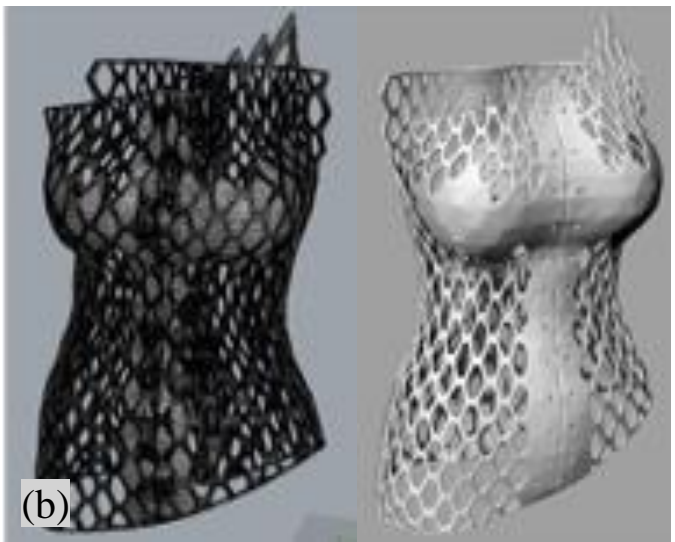

Figure 5. Creation of the 3D model in Rhino®: (a). Hexagon hollows closed at where the drill holes will be and at bust area. (b). The final 3D structure.

Several additional technical challenges arose in the 3D printing phase, namely, the 3D structure was too large for the 3D printer available for this research. This project was printed on a Stratys Fortus 400mc 3D printer with black ABS-M30 ${ }^{\mathrm{TM}}$ material and SR-30 ${ }^{\mathrm{TM}}$ soluble support material. Therefore, it was necessary to cut it one more time horizontally at the waist. Ultimately eight pieces were 3D printed (Figure 6). Geomagic ${ }^{\circledR}$ software was used again to re-render the meshes for improved behavior in printing, and the re-rendering created the change in edge formation along the top and bottom of the structure. These applications reduced the 3D printing

159 time and changed the edge formation along the top and bottom of the structure which 160 subsequently became an unanticipated aesthetic innovation (Figure 6). 

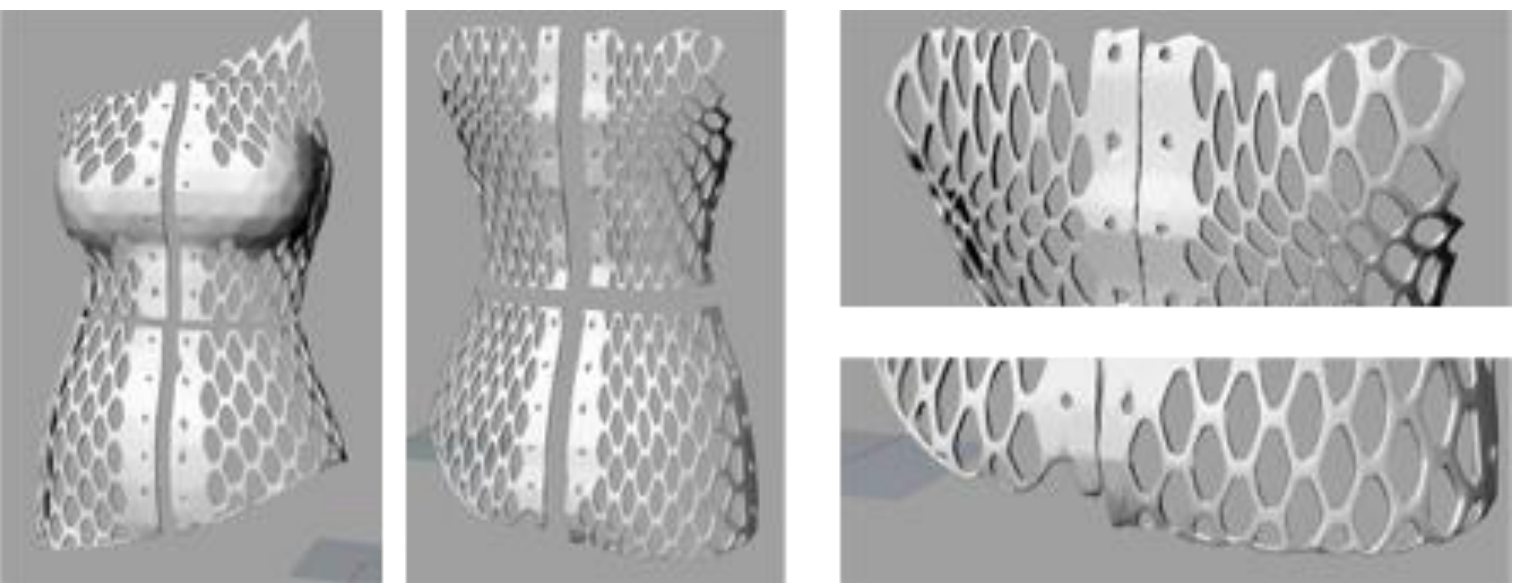

Figure 6. The final 3D pieces that were sent to the 3D printer.

\section{The Customized Pattern Making for the Fabric Layer}

Because corsets are typically covered in fabric to disguise the boning (neither binding tape,

171 create the raw patterns for the scan upon which the 3D corset structure was built. At this point in

172 the process, the patterns were plotted out, digitized into Optitex ${ }^{\mathrm{TM}}$ and exported in the .dxf-aama

173 format, the only format importable to the CLO3D virtual fitting platform. 

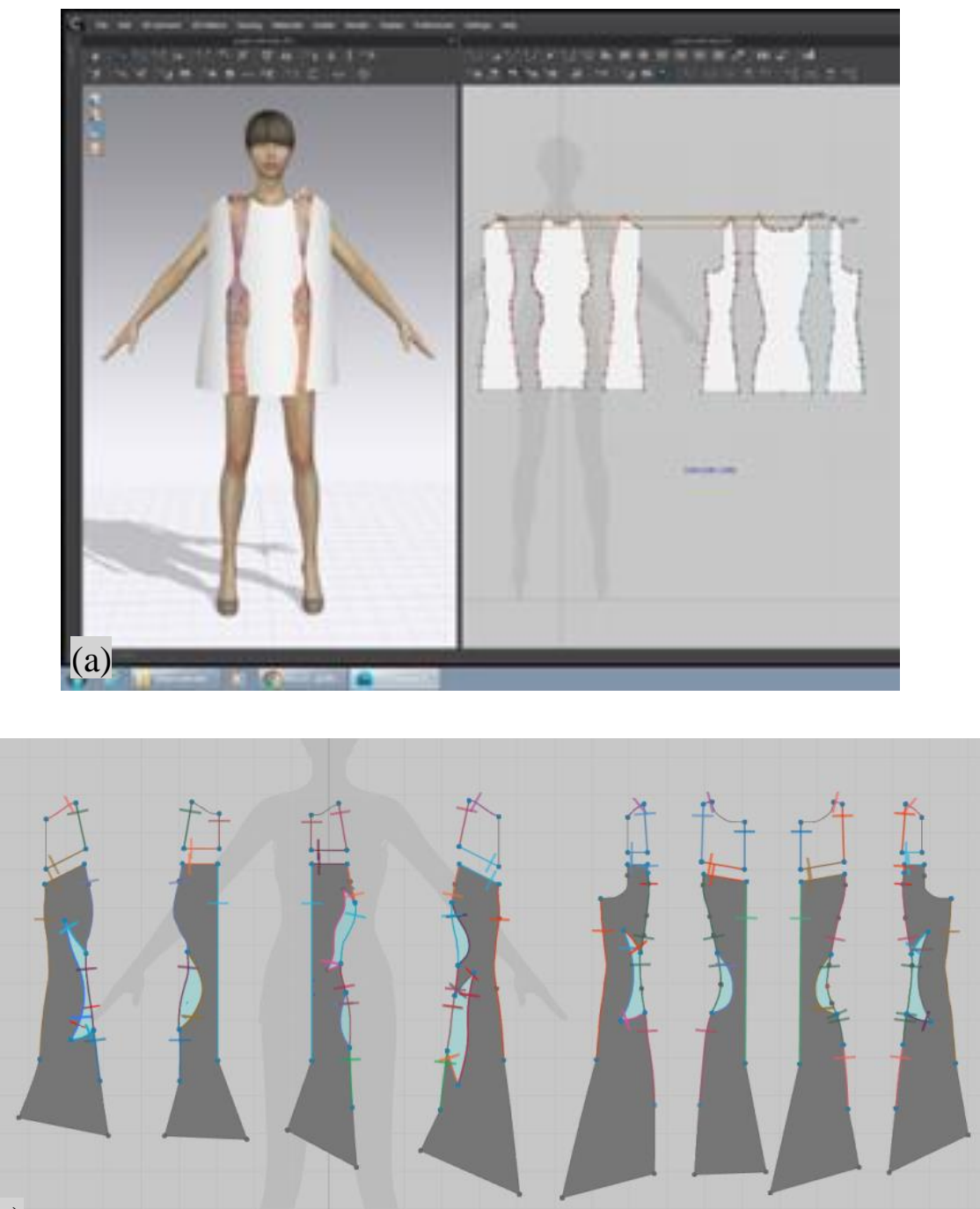

(b)

176 Figure 7. (a) The customized pattern pieces created through Matlab® imported into CLO3D. (b) 177 The final pattern pieces with design elements incorporated.

It has been shown that $3 \mathrm{D}$ virtual try-on technology is an effective way of synthesizing

180 parametric models and offering more realistic visualization (Baytar and Ashdown, 2015; Yu et

181 al., 2012). Using the CLO3D software, it was possible to import the 3D corset structure and

182 place it around the avatar's torso (Figure 7a). It was then necessary to adjust the dimensions of

183 the avatar to fit the shape of the 3D body scan and therefore, to properly test the fit of the pattern

184 pieces. Because CLO3D software cannot guarantee perfect fit, the virtual software was used to

185 make design decisions in a visual format. Given that corsets have historically not had ease, this 
186 contemporary construction similarly did not factor in ease. This was done in attempt to highlight

187 the accuracy of the pattern drafting methods in capturing the shape of the 3D printed structure.

188 One of the greatest design challenges in this project was deciding which design elements to

189 include in in the final artifact. To showcase both designed elements of the final artifact - the 3D

190 printed corset and fabric layer - it was decided to cut decorative shapes into the pattern pieces to

191 draw attention to the 3D printed corset beneath. To create the cut-outs, draft lines were directly

192 drawn on the 3D virtual garments to experiment and come up with the desired shapes. Those

193 draft lines were instantly reflected on the 2D patterns and the pattern pieces were modified based

194 on those draft lines.

195 The finalized patterns (Figure 7b) were exported and converted into an .ai format. Seam

196 allowances were added in Illustrator®. Because it was necessary for the outer layer and the

197 under layer to work in tandem as an overall garment, metal eyelet grommets were used to lace

198 the fabric layer to the 3D corset structure. The holes for lacing were built in to the 3D file in

199 Rhino as shown in Figure 5b. In all instances, the fabric was laser cut to maintain the degree of

200 precision from the final pattern pieces (Figure 7b).

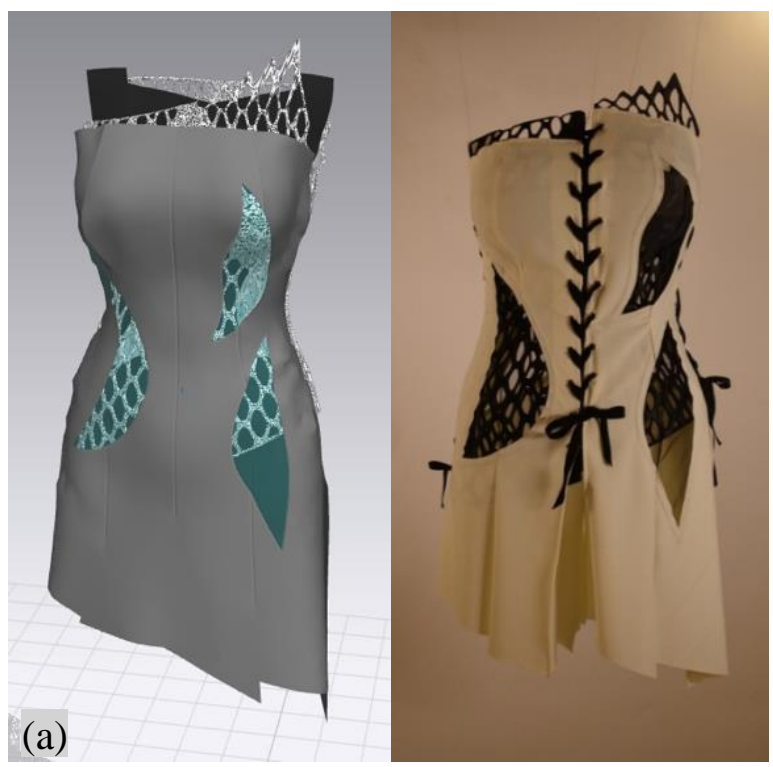




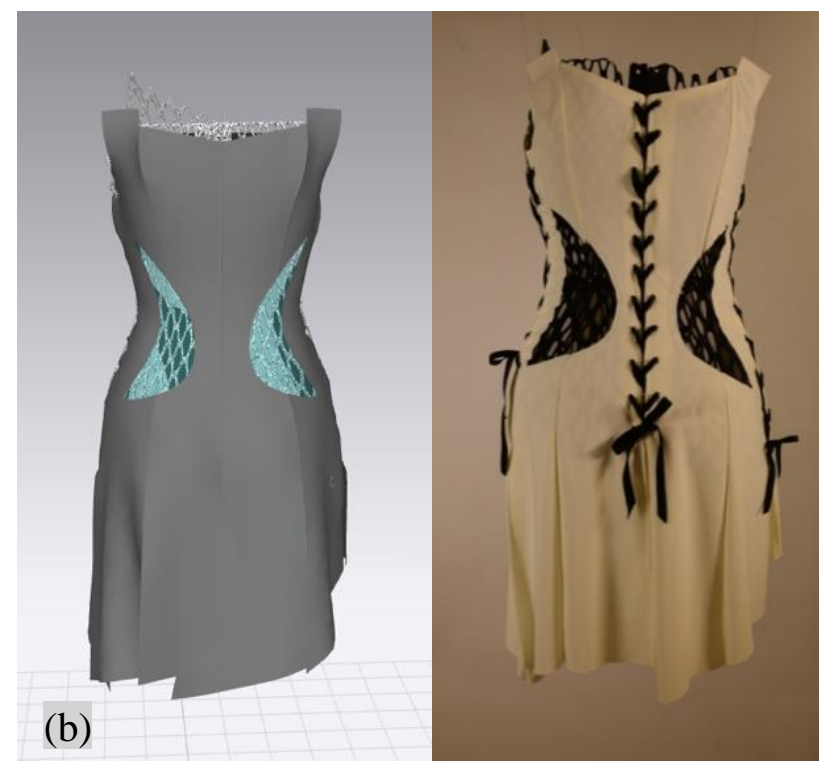

203 Figure 8. (a) The front view and (b) the back view of the final corset and garment.

\section{Discussion and Conclusion}

The methods or protocol-based framework which nucleated this design project enabled

207 more concentrated research into the step-by-step procedure and computational techniques. The

208 final artifact illustrated the efficacy of the design methods. As such, the framework in this project

209 offered potential avenues to further computational design research and build a computational

210 design practice. As a method, the scan-to-pattern process was fast, integrated (the process went

211 from body scan to pattern in one-click), and there was no need for detecting and placing

212 landmarks on the body. The algorithm factored in body shape, body thickness, minor

213 concavity/convexity in contours; and the connectivity of parametric theorems made it possible to

214 manipulate shape in 3D more easily on a computer as opposed to multiple rounds of muslin

215 prototyping. By using body scan data and computer coding, the computational construction

216 methods in this study demonstrate a pliant and sustainable method of clothing design wherein

217 designers were able to use the $\mathrm{X}, \mathrm{Y}$, and $\mathrm{Z}$ planes in differentiating shapes and sizes throughout

218 the construction process. While previous garment construction techniques have relied on the 
219 tailor's skill in translating the 3D shape of the body into 2D patterns and vice versa, the

220 computational methods discussed in this paper enabled work to take place in three dimensions

221 from ideation to creation.

222 In practice, the use of the virtual simulation software (CLO3D) streamlined the

223 prototyping process. Mesh projection uses a series of data points from the 3D body scan which

224 are patched using computer generated algorithms and then easily replicated on other parts of the

225 body. Through the use of parametrics, the data points collected in 3D body scanning enabled the

226 regulation of each aspect of shape manipulation through modifications of the algorithm. The use

227 parametrics aided in balancing interference in the scan's data points. Because each 3D mesh is

228 composed of multiple planes, every aspect of shape manipulation in the $\mathrm{X}, \mathrm{Y}$, and Z coordinates

229 was manipulatable through the use of the computer.

230 The challenges presented in the transition to the final $3 \mathrm{D}$ printing process led to the re-

231 rendering of the parametric design using Mesh Doctor in Geomagic ${ }^{\circledR}$ (Figure 6). The results of

232 these efforts became an unexpected design element and subsequently a source of further

233 research. The irregularities that arose in the transition from software to software were at first

234 viewed as a dilemma, however, upon further research they became integral to the research

235 driving this study. While the design irregularities in this research were not consciously

236 constructed, they served as an important factor in shaping the context and aesthetic of the final

237 designed objects (Figure 9). 


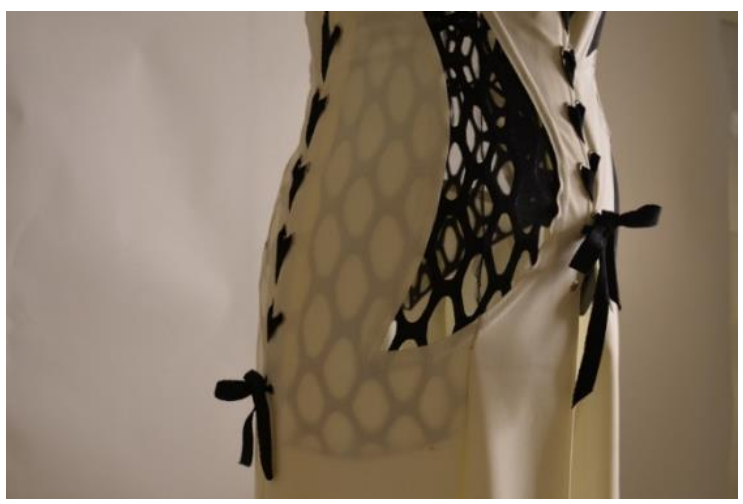

239 Figure 9. The detail view of the final corset and garment.

240 While this study was less concerned with artificial intelligence and the possible uses of

241 machine learning, the construction of patterns using algorithmic design techniques have the

242 potential to be used in approaches which are unscientific and biased. As body scan data and the

243 use of biometric information become increasingly common components of computational

244 fashion design, the ethical use of larger data sets, particularly with the aim to use predictive

245 modeling, should a significant consideration for research agendas. Future directions for this

246 project entail conducting a formal fit test to measure the accuracy of the computational

247 techniques.

248 Through the use of computational design methodology, this study sought to illustrate the

249 use of algorithm driven computer programming to advance creative design practices. As a design

250 tool, a custom-coded garment based on a unique body scan offered a highly malleable method of

251 pattern construction. The corset, the final artifact of this study, was created to explore the vast

252 web of data from a 3D body scan as an expressive way of creating form, shape, and proportion.

253 The process of designing in 3D with the additional use of the z-plane, allowed for the dynamic

254 capability to manipulate proportion and form using parametric design techniques. Further, the

255 use of parametric design in one of the early stages of development allowed for increased

256 flexibility in tackling challenges inherent in the design process. The need for adaptability and 
responsiveness in fashion design is reflective of systematic analysis of daily wear and tear. As

258 such, advancements in design protocol and the creation of a forum for methods-based research is

259 imperative as digital innovation transforms the apparel industry.

\section{References}

Ashdown, S., Loker, S., 2010. Mass-customized Target Market Sizing: Extending the Sizing Paradigm for Improved Apparel Fit. Fashion Practice 2, 147-173.

Ashdown, S., Sung Choi, M., Milke, E., 2008. Automated side-seam placement from 3D body scan data. Int Jnl of Clothing Sci \& Tech 20, 199-213.

Baytar, F., Ashdown, S., 2015. An Exploratory Study of Interaction Patterns around the Use of Virtual Apparel Design and Try-on Technology. Fashion Practice 7, 31-52.

Beck, M., Crié, D., 2018. I virtually try it ... I want it ! Virtual Fitting Room: A tool to increase on-line and off-line exploratory behavior, patronage and purchase intentions. Journal of Retailing and Consumer Services 40, 279-286.

Bye, E., 2010. A Direction for Clothing and Textile Design Research. Clothing and Textiles Research Journal 28, 205-217.

Daanen, H.A.M., Ter Haar, F.B., 2013. 3D whole body scanners revisited. Displays 34, 270-275.

Edelson, D.C.E.R., 2002. Design Research: What We Learn When We Engage in Design. The Journal of the Learning Sciences 11, 105-121.

Griffey, J.V., Ashdown, S.P., 2006. Development of an Automated Process for the Creation of a Basic Skirt Block Pattern from 3D Body Scan Data. Clothing and Textiles Research Journal 24, 112-120.

Kim, J., Forsythe, S., 2008. Adoption of Virtual Try-on technology for online apparel shopping. Journal of Interactive Marketing 22, 45-59.

Kim, S., 2015. Analysis of human body surface shape using parametric design method. Int Jnl of Clothing Sci \& Tech 27, 434-446.

Lee, E., Park, H., 2017. 3D Virtual fit simulation technology: strengths and areas of improvement for increased industry adoption. International Journal of Fashion Design, Technology and Education 10, 59-70.

Lee, Y.-A., 2014. Computer Design and Digital Fit of Clothing, in: Gupta, D., Zakaria, N. (Eds.), Anthropometry, Apparel Sizing and Design. Elsevier Science \& Technology, Cambridge, UNITED KINGDOM, pp. 305-318. 
Lin, Y.-L., Wang, M.-J.J., 2016. The development of a clothing fit evaluation system under virtual environment. Multimed Tools Appl 75, 7575-7587.

Loker, S., Ashdown, S., Carnrite, E., 2008. Dress in the Third Dimension: Online Interactivity and Its New Horizons. Clothing and Textiles Research Journal 26, 164-176.

McKinney, E., Gill, S., Dorie, A., Roth, S., 2017. Body-to-Pattern Relationships in Women's Trouser Drafting Methods: Implications for Apparel Mass Customization. Clothing and Textiles Research Journal 35, 16-32.

Merle, A., Senecal, S., St-Onge, A., 2012. Whether and How Virtual Try-On Influences Consumer Responses to an Apparel Web Site. International Journal of Electronic Commerce 16, 41-64.

Petrova, A., Ashdown, S.P., 2008. Three-Dimensional Body Scan Data Analysis: Body Size and Shape Dependence of Ease Values for Pants' Fit. Clothing and Textiles Research Journal $26,227-252$.

Poole, M., Shvartzberg, Manuel. (Eds.), 2015. The politics of parametricism: digital technologies in architecture. Bloomsbury Academic, London.

Song, H.K., Ashdown, S.P., 2015. Investigation of the Validity of 3-D Virtual Fitting for Pants. Clothing and Textiles Research Journal 33, 314-330.

Verner, A., 2018. Iris van Herpen Fall 2018 Couture Fashion Show [WWW Document]. Vogue. URL https://www.vogue.com/fashion-shows/fall-2018-couture/iris-van-herpen.

Winakor, G., Beck, M.S., Park, S., 1990. Using Geometric Models to Develop a Pattern for the Lower Bodice. Clothing and Textiles Research Journal 8, 49-55.

Yu, H., Qin, S., Sun, G., Wright, D.K., 2012. On generating realistic avatars: dress in your own style. Multimedia Tools and Applications; Dordrecht 59, 973-990. 